CARPATHIAN J. MATH.

Volume 37 (2021), No. 1,

Pages 01 - 11
Online version at https : //www. carpathian. cunbm. utcluj. ro/

Print Edition: ISSN 1584 - 2851; Online Edition: ISSN 1843 - 4401

DOI: https://doi.org/10.37193/CJM.2021.01.01

\title{
General multiplicative Zagreb indices of unicyclic graphs
}

\author{
Monther R. Alfuraidan, Selvaraj Balachandran and Tomáš Vetrík
}

\begin{abstract}
General multiplicative Zagreb indices generalize well-known multiplicative Zagreb indices of graphs. We present lower and upper bounds on the general multiplicative Zagreb indices for unicyclic graphs with given number of vertices and diameter/number of pendant vertices/cycle of given length. All bounds are best possible. Bounds on the classical multiplicative Zagreb indices of unicyclic graphs are corollaries of the general results.
\end{abstract}

\section{INTRODUCTION}

Let $V(G)$ be the vertex set and let $E(G)$ be the edge set of a graph $G$. The order $n$ of a graph $G$ is the number of vertices of $G$. The degree of a vertex $v \in V(G)$, denoted by $d_{G}(v)$, is the number of edges incident with $v$. A pendant vertex is a vertex of degree one. The distance between two vertices is the number of edges in a shortest path connecting them and the diameter of $G$ is the distance between any two farthest vertices in $G$. A diametral path is a shortest path in $G$ connecting two farthest vertices in $G$. A unicyclic graph is a connected graph containing exactly one cycle. We denote the star, the cycle and the path graph of order $n$ by $S_{n}, C_{n}$ and $P_{n}$, respectively.

Multiplicative Zagreb indices have numerous applications and they have been studied especially in the last decade. They play a significant role in chemistry, materials science, pharmaceutical sciences and engineering, since they can be correlated with a large number of physico-chemical properties of molecules. Graph theory is used to characterize these chemical structures. Vertices of graphs correspond to the atoms of a compound and edges of graphs correspond to chemical bonds.

Lower and upper bounds on the multiplicative Zagreb indices for unicyclic graphs of given order were obtained by $\mathrm{Xu}$ and Hua [15], sharp upper bounds for graphs of prescribed order and size were obtained in [7], sharp upper bounds for bipartite graphs of given diameter were given in [11], trees were investigated in [5] and [12], $k$-trees in [14], graphs with a small number of cycles in [1], graph operations in [4] and [8], graphs with cut edges in [13], graphs with given chromatic number in [3], graphs with prescribed clique number in [9], some derived graphs in [2] and molecular graphs in [6].

General multiplicative Zagreb indices were introduced in [10]. The first general multiplicative Zagreb index of a graph $G$ is defined as

$$
P_{1}^{a}(G)=\prod_{v \in V(G)} d_{G}(v)^{a}
$$

and the second general multiplicative Zagreb index is

$$
P_{2}^{a}(G)=\prod_{v \in V(G)} d_{G}(v)^{a d_{G}(v)}=\prod_{u v \in E(G)}\left(d_{G}(u) d_{G}(v)\right)^{a},
$$

Received: 30.04.2019. In revised form: 02.09.2020. Accepted: 07.09.2020

2010 Mathematics Subject Classification. 05C35, 05 C07.

Key words and phrases. multiplicative Zagreb index, unicyclic graph, diameter.

Corresponding author: Selvaraj Balachandran; bala_maths@rediffmail.com 
where $a \neq 0$ is any real number. These indices generalize basic multiplicative Zagreb indices. For $a=1, P_{1}^{1}(G)$ is the Narumi-Katayama index and for $a=2, P_{1}^{2}(G)$ is the first multiplicative Zagreb index

$$
\prod_{1}(G)=\prod_{v \in V(G)} d_{G}(v)^{2} .
$$

For $a=1, P_{2}^{1}(G)$ is the second multiplicative Zagreb index

$$
\prod_{2}(G)=\prod_{v \in V(G)} d_{G}(v)^{d_{G}(v)} .
$$

Let $D=\left(d_{1}, d_{2}, \ldots, d_{n}\right)$ be the degree sequence, where $d_{1} \geq d_{2} \geq \cdots \geq d_{n}$. We introduce

$$
P_{1^{\prime}}^{a}(D)=\prod_{i=1}^{n} d_{i}^{a} \text { and } P_{2^{\prime}}^{a}(D)=\prod_{i=1}^{n} d_{i}^{a d_{i}}
$$

If there is a graph $G$ with vertices having degrees $d_{1}, d_{2}, \ldots, d_{n}$, then the degree sequence $D$ is graphical and we have $P_{1}^{a}(G)=P_{1^{\prime}}^{a}(D)$ and $P_{2}^{a}(G)=P_{2^{\prime}}^{a}(D)$.

It is well-known that for every graph $G, \sum_{v \in V(G)} d_{G}(v)=2 m$, where $m$ is the number of edges of $G$. Since the number of edges is equal to the number of vertices in a unicyclic graph, for a unicyclic graph $G$ we get

$$
\sum_{v \in V(G)} d_{G}(v)=2 n
$$

Unicyclic graphs are interesting, because they can represent various chemical structures. We study unicyclic graphs for the most important parameters. We obtain sharp lower and upper bounds on the general multiplicative Zagreb indices for unicyclic graphs with given number of vertices and diameter/number of pendant vertices/cycle of prescribed length. In all these cases, we characterize the extremal graphs.

\section{PRELIMINARY RESUlTS}

Let us present one lemma and a few corollaries, which will be used in the proofs of our main results.

Lemma 2.1. Let $D$ and $D^{\prime}$ be the degree sequences which differ only in two entries, namely $d_{i}, d_{j}$ are in $D$ and $d_{i}+1, d_{j}-1$ are in $D^{\prime}$, where $d_{i} \geq d_{j} \geq 2$ and $1 \leq i<j \leq n$. Then for $a>0$, $P_{1^{\prime}}^{a}\left(D^{\prime}\right)<P_{1^{\prime}}^{a}(D)$ and $P_{2^{\prime}}^{a}\left(D^{\prime}\right)>P_{2^{\prime}}^{a}(D)$.

Proof. For $a>0$, we have

$$
\frac{P_{1^{\prime}}^{a}(D)}{P_{1^{\prime}}^{a}\left(D^{\prime}\right)}=\frac{d_{i}^{a} d_{j}^{a}}{\left(d_{i}+1\right)^{a}\left(d_{j}-1\right)^{a}}=\left(\frac{d_{i} d_{j}}{d_{i} d_{j}+d_{j}-d_{i}-1}\right)^{a}>1
$$

aince $d_{j}-d_{i}-1<0$. For the $P_{2^{\prime}}^{a}$ index, we have

$$
\begin{aligned}
\frac{P_{2^{\prime}}^{a}(D)}{P_{2^{\prime}}^{a}\left(D^{\prime}\right)} & =\frac{d_{i}^{a d_{i}} d_{j}^{a d_{j}}}{\left(d_{i}+1\right)^{a\left(d_{i}+1\right)}\left(d_{j}-1\right)^{a\left(d_{j}-1\right)}}=\left[\frac{d_{i}^{d_{i}} d_{j}^{d_{j}}}{\left(d_{i}+1\right)^{d_{i}+1}\left(d_{j}-1\right)^{d_{j}-1}}\right]^{a} \\
& =\left[\left(1-\frac{1}{d_{i}+1}\right)^{d_{i}+1}\left(1+\frac{1}{d_{j}-1}\right)^{d_{j}-1} \frac{d_{j}}{d_{i}}\right]^{a}<1,
\end{aligned}
$$

since $\left(1-\frac{1}{d_{i}+1}\right)^{d_{i}+1}<\frac{1}{e},\left(1+\frac{1}{d_{j}-1}\right)^{d_{j}-1}<e$ and $\frac{d_{j}}{d_{i}} \leq 1$. Hence $P_{1^{\prime}}^{a}(D)>P_{1^{\prime}}^{a}\left(D^{\prime}\right)$ and $P_{2^{\prime}}^{a}(D)<P_{2^{\prime}}^{a}\left(D^{\prime}\right)$. 
Corollary 2.1. Let $a>0$ and $D$ be the degree sequence having the smallest $P_{1^{\prime}}^{a}$ index (the largest $P_{2^{\prime}}^{a}$ index) with at most $t \geq 1$ entries equal to 1 , where $\sum_{i=1}^{n} d_{i}=2 n$. Then $D$ has exactly $t$ entries equal to 1 .

Proof. Assume to the contrary that $D=(d_{1}, d_{2}, \ldots, d_{n-t+\epsilon}, \underbrace{1,1, \ldots, 1}_{t-\epsilon})$ where $\epsilon \geq 1$ and $d_{1}, d_{2}, \ldots, d_{n-t+\epsilon} \geq 2$, is the degree sequence having the smallest $P_{1}^{a}$ index (the largest $P_{2^{\prime}}^{a}$ index). From Lemma 2.1 we know that we can replace $d_{1}$ and $d_{n-t+\epsilon}$ by $d_{1}+1$ and $d_{n-t+\epsilon}-1$ to obtain $D^{\prime}=(d_{1}+1, d_{2}, \ldots, d_{n-t+\epsilon}-1, \underbrace{1,1, \ldots, 1}_{t-\epsilon})$ having smaller $P_{1^{\prime}}^{a}$ index (larger $P_{2^{\prime}}^{a}$ index). So $P_{1^{\prime}}^{a}\left(D^{\prime}\right)<P_{1^{\prime}}^{a}(D)$ and $P_{2^{\prime}}^{a}\left(D^{\prime}\right)>P_{2^{\prime}}^{a}(D)$, a contradiction.

Corollary 2.2. Let $a>0$ and $D$ be the degree sequence having the smallest $P_{1^{\prime}}^{a}$ index (the largest $P_{2^{\prime}}^{a}$ index) with $t \geq 0$ entries equal to 1 , where $\sum_{i=1}^{n} d_{i}=2 n$. Then $D=(t+2, \underbrace{2, \ldots, 2}_{n-t-1}, \underbrace{1, \ldots, 1}_{t})$.

Proof. First we prove that the degree sequence $D$ with the smallest $P_{1^{\prime}}^{a}$ index (the largest $P_{2^{\prime}}^{a}$ index) has at most one entry greater than 2 .

Assume to the contrary that $D$ is the degree sequence with the smallest $P_{1^{\prime}}^{a}$ index (the largest $P_{2^{\prime}}^{a}$ index) has at least two entries greater than 2. So $D=(d_{1}, d_{2}, \ldots, d_{s}, \underbrace{2, \ldots, 2}_{n-s-t}$, $\underbrace{1, \ldots, 1}_{t})$, where $s \geq 2$ and $d_{1}, d_{2}, \ldots, d_{s} \geq 3$. By Lemma 2.1, $D^{\prime}=\left(d_{1}+1, d_{2}, \ldots, d_{s}-\right.$ $1, \underbrace{2, \ldots, 2}_{n-s-t}, \underbrace{1, \ldots, 1}_{t}$ ) has smaller $P_{1^{\prime}}^{a}$ index (larger $P_{2^{\prime}}^{a}$ index). So $D$ is not the degree sequence with the smallest $P_{1^{\prime}}^{a}$ index (the largest $P_{2^{\prime}}^{a}$ index). A contradiction.

Thus $D=(d_{1}, \underbrace{2, \ldots, 2}_{n-t-1}, \underbrace{1, \ldots, 1}_{t})$. Since $\sum_{i=1}^{n} d_{i}=d_{1}+2(n-t-1)+t=2 n$, we obtain $d_{1}=t+2$.

Corollary 2.3. Let $a>0$ and $D$ be the degree sequence having the largest $P_{1^{\prime}}^{a}$ index (the smallest $P_{2^{\prime}}^{a}$ index) with $t \geq 0$ entries equal to 1 , where $\sum_{i=1}^{n} d_{i}=2 n$. Then $D=\left(d_{1}, d_{2}, \ldots, d_{n-t}\right.$, $\underbrace{1, \ldots, 1}_{t})$, where $d_{i}-d_{j} \leq 1$ for $1 \leq i<j \leq n-t$.

Proof. Let $D^{\prime}=(d_{1}, d_{2}, \ldots, d_{n-t}, \underbrace{1, \ldots, 1}_{t})$ be the degree sequence having the largest $P_{1^{\prime}}^{a}$ index (the smallest $P_{2^{\prime}}^{a}$ index) with $t$ entries equal to 1 . Since $d_{1} \geq d_{2} \geq \cdots \geq d_{n-t}$, we must show that $d_{1}-d_{n-t} \leq 1$.

Assume to the contrary that $d_{1}-d_{n-t} \geq 2$. Then by Lemma 2.1, a new degree sequence with $d_{1}-1$ and $d_{n-t}+1$ has larger $P_{1^{\prime}}^{a}$ index (smaller $P_{2^{\prime}}^{a}$ index), which is a contradiction.

Corollary 2.4. Let $a>0$ and $D$ be the degree sequence having the largest $P_{1^{\prime}}^{a}$ index (the smallest $P_{2^{\prime}}^{a}$ index), where $\sum_{i=1}^{n} d_{i}=2 n$. Then $D=(2,2, \ldots, 2)$.

Proof. Let $D=\left(d_{1}, d_{2}, \ldots, d_{n}\right)$ be the degree sequence having the largest $P_{1^{\prime}}^{a}$ index (the smallest $P_{2^{\prime}}^{a}$ index). Note that $D$ does not necessarily contain entries 1 . By Corollary 2.3, $d_{i}-d_{j} \leq 1$ for $1 \leq i<j \leq n$. The only sequence satisfying this condition and $\sum_{i=1}^{n} d_{i}=2 n$ is $D=(2,2, \ldots, 2)$. 


\section{UNICYCLIC GRAPHS WITH GIVEN DIAMETER}

Let us study the general multiplicative Zagreb indices for unicyclic graphs with $n$ vertices and diameter $k$. The only graphs having diameter 1 are complete graphs, which are not unicyclic graphs for $n \geq 4$. The only graphs having diameter $n-1$ are paths. So all unicyclic graphs for $n \geq 4$ have diameter $k$, where $2 \leq k \leq n-2$.

The only unicyclic graphs having diameter 2 for $n \geq 6$ consist of the cycle $C_{3}$ and $n-3$ pendant vertices adjacent to one of the vertices of that $C_{3}$. Therefore, we study the case $3 \leq k \leq n-2$.

Let $u_{0} u_{1} \ldots u_{k}$ be a path of order $k+1$ and length $k$, where $k \geq 3$. Let $S_{n, k}$ be the set containing the graphs $U_{n, k, i, \epsilon}$ obtained from the path $u_{0} u_{1} \ldots u_{k}$ by attaching $n-k-1$ new vertices to $u_{i}$ and joining one of the new vertices to $u_{i+\epsilon}$, where $\epsilon=1$ or 2 , and $1 \leq i<i+\epsilon \leq k-1$. We prove that the set $S_{n, k}$ is the family of extremal unicyclic graphs.

Theorem 3.1. Let $G$ be any unicyclic graph having $n$ vertices and diameter $k$, where $3 \leq k \leq$ $n-2$. Then for $a>0$,

$$
P_{1}^{a}(G) \geq(n-k+1)^{a} 3^{a} 2^{a(k-2)} \text { and } P_{2}^{a}(G) \leq(n-k+1)^{(n-k+1) a} 3^{3 a} 2^{2 a(k-2)}
$$

with equalities if and only if $G \in S_{n, k}$.

Proof. Let $G$ be any unicyclic graph having $n$ vertices and diameter $k$. Let $P=u_{0} u_{1} \ldots u_{k}$ be a diametral path in $G$.

Claim 1. $D(G)=(d_{1}, d_{2}, \ldots, d_{k+c}, \underbrace{1,1, \ldots, 1}_{n-k-c})$ where $c \geq 0$.

Clearly, $d_{G}\left(u_{i}\right) \geq 2$ for $i=1,2, \ldots, k-1$. Moreover, the cycle of $G$ must contain at least one vertex which is not in $P$. This implies that $G$ contains at least $k$ vertices of degree greater than 1 and at most $n-k$ vertices of degree 1 .

Claim 2. No unicyclic graph has the degree sequence $(n-k+2, \underbrace{2,2, \ldots, 2}_{k-1}, \underbrace{1,1, \ldots, 1}_{n-k})$. Assume to the contrary that $D(G)=(n-k+2, \underbrace{2,2, \ldots, 2}_{k-1}, \underbrace{1,1, \ldots, 1}_{n-k})$. Obviously, $k-1$ vertices of the diametral path $u_{1}, u_{2}, \ldots, u_{k-1}$ have degree at least 2 . Let $u^{\prime}$ be the $k$ th vertex of degree at least 2. All the other vertices have degree 1 . So $u^{\prime}$ must be in the cycle, which means that it is adjacent to 2 vertices, say $u_{i}, u_{j}$ where $i<j$, from the set $\left\{u_{1}, u_{2}, \ldots, u_{k-1}\right\}$. Clearly, $u^{\prime}$ is neither $u_{0}$ not $u_{k}$, otherwise if, say $u^{\prime}=u_{0}$, then $u_{0} u_{j} u_{j+1} \ldots u_{k}$ would be a shorter path than $P$. Then $u_{i}$ and $u_{j}$ have degree at least 3 , which is a contradiction since $D(G)$ has only one entry greater than 2 .

By Claim 1, the degree sequence $D^{\prime}$ having the smallest $P_{1^{\prime}}^{a}$ index (the largest $P_{2^{\prime}}^{a}$ index) satisfying $\sum_{i=1}^{n} d_{i}=2 n$ has at most $n-k$ entries equal to 1 . Then by Corollary 2.1, $D^{\prime}$ has exactly $n-k$ entries equal to 1 . From Corollary 2.2 we know that $D^{\prime}$ is the sequence given in Claim 2. However, there is no unicyclic graph of diameter $k$ having that degree sequence. By Lemma 2.1, the degree sequence having the second smallest $P_{1}^{a}$ index (the second largest $P_{2^{\prime}}^{a}$ index) satisfying $\sum_{i=1}^{n} d_{i}=2 n$ and Claim 1 is the sequence $(n-k+$ $1,3, \underbrace{2,2, \ldots, 2}_{k-2}, \underbrace{1,1, \ldots, 1}_{n-k})$.

Let us find all graphs $G^{\prime}$ having that degree sequence. Clearly, the vertices $u_{1}$, $u_{2}, \ldots, u_{k-1}$ of the diametral path have degree at least 2 . Let us denote the set of these vertices by $U$. Since $G^{\prime}$ has a cycle and exactly $k$ vertices of degree at least $2, G^{\prime}$ contains one vertex, say $x$, connected to 2 vertices of $U$, say $u_{i}$ and $u_{j}$. So, $u_{i}$ and $u_{j}$ are of distance at most 2 . Thus $j \leq i+2$. Since $G^{\prime}$ has a vertex of degree $n-k+1$ and only one vertex of degree 3 , it follows that one of $u_{i}, u_{j}$ is adjacent to $n-k-2$ pendant vertices 
$x_{1}, x_{2}, \ldots, x_{n-k-2}$. The other two pendant vertices are $u_{0}$ and $u_{k}$. Hence, $G^{\prime}$ is one of the graphs in $S_{n, k}$. It is easy to see that

$$
P_{1}^{a}\left(G^{\prime}\right)=(n-k+1)^{a} 3^{a} 2^{a(k-2)} \text { and } P_{2}^{a}\left(G^{\prime}\right)=(n-k+1)^{(n-k+1) a} 3^{3 a} 2^{2 a(k-2)},
$$

thus the proof is complete.

For $z \geq 3$ and $b \geq 0$, let $C_{z} \star P_{b+1}$ be a graph obtained by identifying one vertex of the cycle $C_{z}$ with the end vertex of the path $P_{b+1}$. Let us show that $C_{2 n-2 k} \star P_{2 k-n+1}$ and $C_{2 n-2 k-1} \star P_{2 k-n+2}$ are the only unicyclic graphs of order $n$ and diameter $k$ having the largest $P_{1}^{a}$ index and the smallest $P_{2}^{a}$ index for $k>\left\lfloor\frac{n}{2}\right\rfloor$.

Theorem 3.2. Let $a>0$ and let $G$ be any unicyclic graph having $n$ vertices and diameter $k$. If $\left\lfloor\frac{n}{2}\right\rfloor<k \leq n-2$, then

$$
P_{1}^{a}(G) \leq 3^{a} 2^{a(n-2)} \text { and } P_{2}^{a}(G) \geq 3^{3 a} 2^{2 a(n-2)},
$$

with equalities if and only if $G$ is $C_{2 n-2 k} \star P_{2 k-n+1}$ or $C_{2 n-2 k-1} \star P_{2 k-n+2}$. If $k=\left\lfloor\frac{n}{2}\right\rfloor$, then

$$
P_{1}^{a}(G) \leq 2^{a n} \text { and } P_{2}^{a}(G) \geq 2^{2 a n},
$$

with equalities if and only if $G$ is $C_{n}$.

Proof. Let $G$ be a unicyclic graph having $n$ vertices and diameter $k$ with the largest $P_{1}^{a}$ index (the smallest $P_{2}^{a}$ index)

From Corollary 2.4 we know that the degree sequence having the largest $P_{1}^{a}$ index (the smallest $P_{2^{\prime}}^{a}$ index) is $(2,2, \ldots, 2)$. The only (connected) graph having this degree sequence is $C_{n}$, which has the diameter $\left\lfloor\frac{n}{2}\right\rfloor$. Obviously, $P_{1}^{a}\left(C_{n}\right)=2^{a n}$ and $P_{2}^{a}\left(C_{n}\right)=2^{2 a n}$.

So we consider the problem for $\left\lfloor\frac{n}{2}\right\rfloor<k \leq n-2$. The degree sequence having the second largest $P_{1^{\prime}}^{a}$ index (the second smallest $P_{2^{\prime}}^{a}$ index) is $(3, \underbrace{2, \ldots, 2}_{n-2}, 1)$. It is easy to check that the only unicyclic graph having this sequence is $C_{z} \star P_{b+1}$. Since this graph is obtained by identifying one vertex of $C_{z}$ with the end vertex of $P_{b}$, the order $n=z+b$. The diameter of $C_{z} \star P_{b+1}$ is $k=\left\lfloor\frac{z}{2}\right\rfloor+b$. We express $z$ and $b+1$ in terms of $n$ and $k$. Since

$$
n=z+b \text { and } k=\left\lfloor\frac{z}{2}\right\rfloor+b, \text { we get } n-k=\left\lceil\frac{z}{2}\right\rceil .
$$

This implies that if $z$ is even, then

$$
z=2 n-2 k \text { and } b=n-z=2 k-n .
$$

If $a$ is odd, then

$$
z=2 n-2 k-1 \text { and } b=n-z=2 k-n+1 .
$$

Hence, $C_{2 n-2 k} \star P_{2 k-n+1}$ and $C_{2 n-2 k-1} \star P_{2 k-n+2}$ are the only graphs having the largest $P_{1}^{a}$ index (the smallest $P_{2}^{a}$ index) for $k>\left\lfloor\frac{n}{2}\right\rfloor$.

We have

$$
P_{1}^{a}\left(C_{2 n-2 k} \star P_{2 k-n+1}\right)=P_{1}^{a}\left(C_{2 n-2 k-1} \star P_{2 k-n+2}\right)=3^{a} 2^{a(n-2)}
$$

and

$$
P_{2}^{a}\left(C_{2 n-2 k} \star P_{2 k-n+1}\right)=P_{2}^{a}\left(C_{2 n-2 k-1} \star P_{2 k-n+2}\right)=3^{3 a} 2^{2 a(n-2)},
$$

which completes the proof. 


\section{UNICYCLIC GRAPHS WITH GIVEN NUMBER OF PENDANT VERTICES}

In this section we give lower and upper bounds on the general multiplicative Zagreb indices for unicyclic graphs with given number of vertices and number of pendant vertices. Since every unicyclic graph contains a cycle having at least 3 vertices, those vertices are not pendant. Thus the number $p$ of pendant vertices in a unicyclic graph is $0 \leq p \leq n-3$.

Let $H$ be a unicyclic graph containing the cycle $C_{3}$, where one of the vertices of the cycle is adjacent to $p$ pendant vertices and let $U_{p+3, p}=\{H\}$. Let $U_{p+i+1, p}$ be the set of unicyclic graphs containing all graphs obtained by the subdivision of any edge of every graph in $U_{p+i, p}$, where $i=3,4, \ldots, n-p-1$. We show that $U_{n, p}$ is the set of extremal unicyclic graphs with respect to the order and the number of pendant vertices

Theorem 4.3. Let $G$ be any unicyclic graph having $n$ vertices and $p$ pendant vertices, where $0 \leq p \leq n-3$. Then for $a>0$,

$$
P_{1}^{a}(G) \geq(p+2)^{a} 2^{a(n-p-1)} \text { and } P_{2}^{a}(G) \leq(p+2)^{a(p+2)} 2^{2 a(n-p-1)}
$$

with equalities if and only if $G$ is in $U_{n, p}$.

Proof. Let $G$ be any unicyclic graph having $n$ vertices and $p$ pendant vertices. Obviously, $G$ has exactly $p$ vertices of degree 1 . By Corollary 2.2, the degree sequence having the smallest $P_{1^{\prime}}^{a}$ index (the largest $P_{2^{\prime}}^{a}$ index) is $(d_{1}, \underbrace{2, \ldots, 2}_{n-p-1}, \underbrace{1, \ldots, 1}_{p})$, where $d_{1}=p+2$.

The only graphs having the degree sequence $(p+2, \underbrace{2, \ldots, 2}_{n-p-1}, \underbrace{1, \ldots, 1}_{p})$ are the graphs in the set $U_{n, p}$. Thus the graphs $G^{\prime} \in U_{n, p}$ are the unicyclic graphs having the smallest $P_{1}^{a}$ index (the largest $P_{2}^{a}$ index). We obtain

$$
P_{1}^{a}\left(G^{\prime}\right)=(p+2)^{a} 2^{a(n-p-1)} \text { and } P_{2}^{a}\left(G^{\prime}\right)=(p+2)^{a(p+2)} 2^{2 a(n-p-1)} .
$$

Now we obtain a sharp upper bound on $P_{1}^{a}(G)$ and a sharp lower bound on $P_{2}^{a}(G)$, where $G$ is a unicyclic graph of given order and number of pendant vertices.

Theorem 4.4. Let $G$ be any unicyclic graph having $n$ vertices and $p$ pendant vertices, where $0 \leq p \leq n-3$. Then for $a>0$,

$$
P_{1}^{a}(G) \leq(b+2)^{a[n-(n-p) b]}(b+1)^{a[(n-p) b-p]}
$$

and

$$
P_{2}^{a}(G) \geq(b+2)^{a(b+2)[n-(n-p) b]}(b+1)^{a(b+1)[(n-p) b-p]}
$$

with equalities if and only if $G$ has the degree sequence $(\underbrace{b+2, \ldots, b+2}_{n-(n-p) b}, \underbrace{b+1, \ldots, b+1}_{(n-p) b-p}, \underbrace{1, \ldots, 1}_{p})$,

where $b=\left\lfloor\frac{n}{n-p}\right\rfloor$.

Proof. Let $G$ be any unicyclic graph with $n$ vertices and $p$ pendant vertices. So, $G$ has $p$ vertices of degree 1. By Corollary 2.3, the degree sequence having the largest $P_{1^{\prime}}^{a}$ index (the smallest $P_{2^{\prime}}^{a}$ index) is $D=(d_{1}, d_{2}, \ldots, d_{n-p}, \underbrace{1, \ldots, 1}_{p})$, where $d_{1}-d_{n-p} \leq 1$.

So we can assume that $d_{i}=c$ or $c+1$ for $i=1,2, \ldots, n-p$ and $c \geq 2$. Let $n_{j}$ be the number of entries in $D$ being $j$. Then

$$
D=(\underbrace{c+1, \ldots, c+1}_{n_{c+1}}, \underbrace{c, \ldots, c}_{n_{c}}, \underbrace{1, \ldots, 1}_{p})
$$


and thus

$$
p+n_{c}+n_{c+1}=n .
$$

Clearly, $p<n$, therefore we can assume that $n_{c} \geq 1$. By (1.1),

$$
\sum_{i=1}^{n} d_{i}=p+c n_{c}+(c+1) n_{c+1}=2 n .
$$

By (4.2) and (4.3), we obtain $c p+n_{c}=c n-n$, which gives $c=\frac{n}{n-p}+\frac{n_{c}}{n-p}$. From (4.2) we have $n_{c} \leq n-p$, therefore $c=\left\lfloor\frac{n}{n-p}\right\rfloor+1$, so

$$
n_{c}=(n-p) c-n=(n-p)\left\lfloor\frac{n}{n-p}\right\rfloor-p \text { and } n_{c+1}=n-(n-p)\left\lfloor\frac{n}{n-p}\right\rfloor .
$$

Thus

$$
D=(\underbrace{b+2, \ldots, b+2}_{n-(n-p) b}, \underbrace{b+1, \ldots, b+1}_{(n-p) b-p}, \underbrace{1, \ldots, 1}_{p}),
$$

where $b=\left\lfloor\frac{n}{n-p}\right\rfloor$.

We show that there is a graph $G^{\prime}$ having the degree sequence $D$. Let $G^{\prime}$ be a graph which contains the cycle of length $n-p ; n-(n-p) b$ vertices of the cycle are adjacent to $b$ pendant vertices and the other $(n-p) b-p$ vertices of the cycle are adjacent to $b-1$ pendant vertices, where $b=\left\lfloor\frac{n}{n-p}\right\rfloor$. We get

$$
P_{1}^{a}\left(G^{\prime}\right)=(b+2)^{a[n-(n-p) b]}(b+1)^{a[(n-p) b-p]}
$$

and

$$
P_{2}^{a}\left(G^{\prime}\right)=(b+2)^{a(b+2)[n-(n-p) b]}(b+1)^{a(b+1)[(n-p) b-p]} .
$$

\section{UNICYCLIC GRAPHS WITH GIVEN $k$-CYCLE}

We study unicyclic graphs with a cycle of length $k$. These graphs are also called unicyclic graphs with girth $k$. Clearly, $3 \leq k \leq n$.

Let $C_{k} * S_{n-k+1}$ be the graph obtained by identifying one vertex of the cycle $C_{k}$ with the central vertex of the star $S_{n-k+1}$. We show that $C_{k} * S_{n-k+1}$ has the smallest $P_{1}^{a}$ and the largest $P_{2}^{a}$ among unicyclic graphs of order $n$ which have a $k$-cycle.

Theorem 5.5. Let $G$ be any unicyclic graph having $n$ vertices and a cycle of length $k$, where $3 \leq k \leq n$. Then for $a>0$,

$$
P_{1}^{a}(G) \geq(n-k+2)^{a} 2^{a(k-1)} \text { and } P_{2}^{a}(G) \leq(n-k+2)^{a(n-k+2)} 2^{2 a(k-1)}
$$

with equalities if and only if $G$ is $C_{k} * S_{n-k+1}$.

Proof. Let $G$ be any unicyclic graph having $n$ vertices and a cycle of length $k$. All vertices of the $k$-cycle have degree at least 2 , so $G$ has at most $n-k$ vertices of degree 1 .

By Corollary 2.1, the degree sequence having the smallest $P_{1^{\prime}}^{a}$ index (the largest $P_{2^{\prime}}^{a}$ index) with at most $n-k$ entries being 1 is $(d_{1}, d_{2}, \ldots, d_{k}, \underbrace{1,1, \ldots, 1}_{n-k})$, and then by Corollary 2.2, $d_{2}=d_{3}=\cdots=d_{k}=2$ and $d_{1}=n-k+2$.

The only graph having the degree sequence $(n-k+2, \underbrace{2,2, \ldots, 2}_{k-1}, \underbrace{1,1, \ldots, 1}_{n-k})$ is $C_{k} *$ $S_{n-k+1}$. So $C_{k} * S_{n-k+1}$ is the graph having the smallest $P_{1}^{a}$ index (the largest $P_{2}^{a}$ index). We obtain

$$
P_{1}^{a}\left(C_{k} * S_{n-k+1}\right)=(n-k+2)^{a} 2^{a(k-1)}
$$


and

$$
P_{2}^{a}\left(C_{k} * S_{n-k+1}\right)=(n-k+2)^{(n-k+2) a} 2^{2 a(k-1)} .
$$

Note that $C_{k} \star P_{n-k+1}$ is the graph obtained by identifying one vertex of the cycle $C_{k}$ with the end vertex of the path $P_{n-k+1}$. The graph $C_{k} \star P_{n-k+1}$ has the largest $P_{1}^{a}$ index and the smallest $P_{1}^{a}$ index among unicyclic graphs of order $n$ having a $k$-cycle. Since for $k=n, C_{n}$ is the only graph having a $k$-cycle, we present the bounds for $k<n$.

Theorem 5.6. Let $a>0$ and let $G$ be any unicyclic graph having $n$ vertices and a cycle of length $k$, where $3 \leq k \leq n-1$. Then

$$
P_{1}^{a}(G) \leq 3^{a} 2^{a(n-2)} \text { and } P_{2}^{a}(G) \geq 3^{3 a} 2^{2 a(n-2)},
$$

with equalities if and only if $G$ is $C_{k} \star P_{n-k+1}$.

Proof. Let $G^{\prime}$ be a unicyclic graph having $n$ vertices and a cycle of length $k$ with the largest $P_{1}^{a}$ index (the smallest $P_{2}^{a}$ index). By Corollary 2.4, the degree sequence having the largest $P_{1^{\prime}}^{a}$ index (the smallest $P_{2^{\prime}}^{a}$ index) is $(2,2 \ldots, 2)$. The only (connected) graph having this degree sequence is $C_{n}$, which has the cycle of length $k=n$. So if $k=n$, then $G^{\prime}=C_{n}$. Obviously, $P_{1}^{a}\left(C_{n}\right)=2^{\text {an }}$ and $P_{2}^{a}\left(C_{n}\right)=2^{2 a n}$.

So we consider the problem for $k<n$. The degree sequence having the second largest $P_{1^{\prime}}^{a}$ index (the second smallest $P_{2^{\prime}}^{a}$ index) is $(3, \underbrace{2, \ldots, 2}_{n-2}, 1)$. The only unicyclic graph having this sequence is $C_{k} \star P_{n-k+1}$. Thus $G^{\prime}=C_{k} \star P_{n-k+1}$ is the only graph having the largest $P_{1}^{a}$ index (the smallest $P_{2}^{a}$ index) for $k<n$. We have

$$
P_{1}^{a}\left(C_{k} \star P_{n-k+1}\right)=3^{a} 2^{a(n-2)} \text { and } P_{2}^{a}\left(C_{k} \star P_{n-k+1}\right)=3^{3 a} 2^{2 a(n-2)},
$$

hence the proof is complete.

\section{CONCLUSION}

In Sections 3, 4 and 5 we presented lower and upper bounds on the general multiplicative Zagreb indices for unicyclic graphs with given number of vertices and diameter/number of pendant vertices/ $k$-cycle, where $a>0$.

If $a=2$, then $P_{1}^{2}(G)$ is the first multiplicative Zagreb index $\prod_{1}(G)$, and if $a=1$, then $P_{2}^{1}(G)$ is the second multiplicative Zagreb index $\prod_{2}(G)$. From Theorems $3.1-5.6$ we get the following corollaries.

Corollary 6.5. Let $G$ be any unicyclic graph having $n$ vertices and diameter $k$, where $3 \leq k \leq$ $n-2$. Then

$$
\prod_{1}(G) \geq 9(n-k+1)^{2} 4^{k-2} \text { and } \prod_{2}(G) \leq 27(n-k+1)^{n-k+1} 4^{k-2} .
$$

Corollary 6.6. Let $G$ be any unicyclic graph having $n$ vertices and diameter $k$. If $\left\lfloor\frac{n}{2}\right\rfloor<k \leq n-2$, then

$$
\prod_{1}(G) \leq 9 \cdot 4^{n-2} \text { and } \prod_{2}(G) \geq 27 \cdot 4^{n-2},
$$

with equalities if and only if $G$ is $C_{2 n-2 k} \star P_{2 k-n+1}$ or $C_{2 n-2 k-1} \star P_{2 k-n+2}$. If $k=\left\lfloor\frac{n}{2}\right\rfloor$, then

$$
\prod_{1}(G) \leq 4^{n} \text { and } \prod_{2}(G) \geq 4^{n},
$$

with equalities if and only if $G$ is $C_{n}$. 
Corollary 6.7. Let $G$ be any unicyclic graph having $n$ vertices and $p$ pendant vertices, where $0 \leq p \leq n-3$. Then

$$
(p+2)^{2} 4^{n-p-1} \leq \prod_{1}(G) \leq(b+2)^{2[n-(n-p) b]}(b+1)^{2[(n-p) b-p]},
$$

where $b=\left\lfloor\frac{n}{n-p}\right\rfloor$.

Corollary 6.8. Let $G$ be any unicyclic graph having $n$ vertices and a cycle of length $k$, where $3 \leq k \leq n-1$. Then

$$
(n-k+2)^{2} 4^{k-1} \leq \prod_{1}(G) \leq 9 \cdot 4^{n-2} .
$$

Corollary 6.9. Let $G$ be any unicyclic graph having $n$ vertices and p pendant vertices, where $0 \leq p \leq n-3$. Then

$$
(b+2)^{(b+2)[n-(n-p) b]}(b+1)^{(b+1)[(n-p) b-p]} \leq \prod_{2}(G) \leq(p+2)^{p+2} 4^{n-p-1},
$$

where $b=\left\lfloor\frac{n}{n-p}\right\rfloor$.

Corollary 6.10. Let $G$ be any unicyclic graph having $n$ vertices and a cycle of length $k$, where $3 \leq k \leq n-1$. Then

$$
27 \cdot 4^{n-2} \leq \prod_{2}(G) \leq(n-k+2)^{n-k+2} 4^{k-1} .
$$

In the proof of Lemma 2.1 we use the following: if $x>1$ and $a>0$, then $x^{a}>1$. Similarly, if $0<x<1$ and $a>0$, then $0<x^{a}<1$. We can obtain a lemma for $a<0$ using a similar proof and these facts:

- if $x>1$ and $a<0$, then $0<x^{a}<1$,

- if $0<x<1$ and $a<0$, then $x^{a}>1$.

Lemma 6.2. Let $D$ and $D^{\prime}$ be the degree sequences which differ only in two entries, namely $d_{i}, d_{j}$ are in $D$ and $d_{i}+1, d_{j}-1$ are in $D^{\prime}$, where $d_{i} \geq d_{j} \geq 2$ and $1 \leq i<j \leq n$. Then for $a<0$, $P_{1^{\prime}}^{a}\left(D^{\prime}\right)>P_{1^{\prime}}^{a}(D)$ and $P_{2^{\prime}}^{a}\left(D^{\prime}\right)<P_{2^{\prime}}^{a}(D)$.

Then we can use Lemma 6.2 and corollaries similar to Corollaries 2.1, 2.2, 2.3 and 2.4 to obtain bounds for unicyclic graphs, where $a<0$. Bounds on the general multiplicative Zagreb indices for unicyclic graphs of given order and diameter, where $a<0$, are given in Theorems 6.7 and 6.8 .

Theorem 6.7. Let $G$ be any unicyclic graph having $n$ vertices and diameter $k$, where $3 \leq k \leq$ $n-2$. Then for $a<0$,

$$
P_{1}^{a}(G) \leq(n-k+1)^{a} 3^{a} 2^{a(k-2)} \text { and } P_{2}^{a}(G) \geq(n-k+1)^{(n-k+1) a} 3^{3 a} 2^{2 a(k-2)}
$$

with equalities if and only if $G \in S_{n, k}$.

Theorem 6.8. Let $a<0$ and let $G$ be any unicyclic graph having $n$ vertices and diameter $k$. If $\left\lfloor\frac{n}{2}\right\rfloor<k \leq n-2$, then

$$
P_{1}^{a}(G) \geq 3^{a} 2^{a(n-2)} \text { and } P_{2}^{a}(G) \leq 3^{3 a} 2^{2 a(n-2)},
$$

with equalities if and only if $G$ is $C_{2 n-2 k} \star P_{2 k-n+1}$ or $C_{2 n-2 k-1} \star P_{2 k-n+2}$. If $k=\left\lfloor\frac{n}{2}\right\rfloor$, then

$$
P_{1}^{a}(G) \geq 2^{a n} \text { and } P_{2}^{a}(G) \leq 2^{2 a n},
$$

with equalities if and only if $G$ is $C_{n}$.

Results on unicyclic graphs of given order and number of pendant vertices, where $a<0$, are presented in Theorems 6.9 and 6.10 . 
Theorem 6.9. Let $G$ be any unicyclic graph having $n$ vertices and $p$ pendant vertices, where $0 \leq p \leq n-3$. Then for $a<0$,

$$
P_{1}^{a}(G) \leq(p+2)^{a} 2^{a(n-p-1)} \text { and } P_{2}^{a}(G) \geq(p+2)^{a(p+2)} 2^{2 a(n-p-1)}
$$

with equalities if and only if $G$ is in $U_{n, p}$.

Theorem 6.10. Let $G$ be any unicyclic graph having $n$ vertices and $p$ pendant vertices, where $0 \leq p \leq n-3$. Then for $a<0$,

$$
P_{1}^{a}(G) \geq(b+2)^{a[n-(n-p) b]}(b+1)^{a[(n-p) b-p]}
$$

and

$$
P_{2}^{a}(G) \leq(b+2)^{a(b+2)[n-(n-p) b]}(b+1)^{a(b+1)[(n-p) b-p]}
$$

with equalities if and only if $G$ has the degree sequence $(\underbrace{b+2, \ldots, b+2}_{n-(n-p) b}, \underbrace{b+1, \ldots, b+1}_{(n-p) b-p}, \underbrace{1, \ldots, 1}_{p})$,

where $b=\left\lfloor\frac{n}{n-p}\right\rfloor$.

Finally, we give bounds for unicyclic graphs having a $k$-cycle.

Theorem 6.11. Let $G$ be any unicyclic graph having $n$ vertices and a cycle of length $k$, where $3 \leq k \leq n$. Then for $a<0$,

$$
P_{1}^{a}(G) \leq(n-k+2)^{a} 2^{a(k-1)} \text { and } P_{2}^{a}(G) \geq(n-k+2)^{a(n-k+2)} 2^{2 a(k-1)}
$$

with equalities if and only if $G$ is $C_{k} * S_{n-k+1}$.

Theorem 6.12. Let $a<0$ and let $G$ be any unicyclic graph having $n$ vertices and a cycle of length $k$, where $3 \leq k \leq n-1$. Then

$$
P_{1}^{a}(G) \geq 3^{a} 2^{a(n-2)} \text { and } P_{2}^{a}(G) \leq 3^{3 a} 2^{2 a(n-2)},
$$

with equalities if and only if $G$ is $C_{k} \star P_{n-k+1}$.

Acknowledgments. M. R. Alfuraidan and T. Vetrík acknowledge the support provided by the Deanship of scientific research at King Fahd University of Petroleum and Minerals for funding this work through project No. IN171003.

\section{REFERENCES}

[1] Alfuraidan, M. R., Vetrík, T. and Balachandran, S., General multiplicative Zagreb indices of graphs with a small number of cycles, Symmetry, 12 (2020), No. 4, 514

[2] Basavanagoud, B. and Patil, S., Multiplicative Zagreb indices and coindices of some derived graphs, Opuscula Math., 36 (2016), No. 3, 287-299

[3] Ghorbani, M. and Songhori, M., Computing multiplicative Zagreb indices with respect to chromatic and clique numbers, Iran. J. Math. Chem., 5 (2014), No. 1, 11-18

[4] Das, K. C., Yurttas, A., Togan, M., Cevik, A. S. and Cangul, I. N., The multiplicative Zagreb indices of graph operations, J. Inequal. Appl., 2013, 90 (2013), https:/ / doi.org/10.1186/1029-242X-2013-90

[5] Gutman, I., Multiplicative Zagreb indices of trees, Bull. Internat. Math. Virt. Inst. 1 (2011), 13-19

[6] Kazemi, R., Note on the multiplicative Zagreb indices, Discrete Appl. Math., 198 (2016), 147-154

[7] Liu, J. and Zhang, Q., Sharp upper bounds for multiplicative Zagreb indices, MATCH Commun. Math. Comput. Chem., 68 (2012), No. 1, 231-240

[8] Nezhad, E. F., Iranmanesh, A., Tehranian, A. and Azari, M., Strict lower bounds on the multiplicative Zagreb indices of graph operations, Ars Combin., 117 (2014), 399-409

[9] Vetrík, T. and Balachandran, S., General multiplicative Zagreb indices of graphs with given clique number, Opuscula Math., 39 (2019), No. 3, 433-446

[10] Vetrík, T. and Balachandran, S., General multiplicative Zagreb indices of trees, Discrete Appl. Math., 247 (2018), 341-351

[11] Wang, C., Liu J.-B. and Wang, S., Sharp upper bounds for multiplicative Zagreb indices of bipartite graphs with given diameter, Discrete Appl. Math., 227 (2017), 156-165 
[12] Wang, S., Wang, C., Chen, L. and Liu, J.-B., On extremal multiplicative Zagreb indices of trees with given number of vertices of maximum degree, Discrete Appl. Math., 227 (2017), 166-173

[13] Wang, S., Wang, C., Chen, L., Liu, J.-B. and Shao, Z., Maximizing and minimizing multiplicative Zagreb indices of graphs subject to given number of cut edges, Mathematics, 6 (2018), 227

[14] Wang, S. and Wei, B., Multiplicative Zagreb indices of k-trees, Discrete Appl. Math., 180 (2015), 168-175

[15] Xu, K. and Hua, H., A unified approach to extremal multiplicative Zagreb indices for trees, unicyclic and bicyclic graphs, MATCH Commun. Math. Comput. Chem., 68 (2012), 241-256

DepartMENT OF MATHEMATics AND STATISTics

King FAHD University of Petroleum AND Minerals

DHAHRAN 31261, SAUDI ARABIA

Email address: montherekfupm. edu. sa

Department of Mathematics and Applied Mathematics

UNIVERSITY OF THE FREE STATE

P. O. Box 339, 9300, BLOEMFONTEIN, SOUTH AFricA

AND

Department of Mathematics, School of ARTS, SCIENCES AND Humanities

SASTRA DEEMED UNIVERSITY

THANJAVUR, INDIA

Email address: bala_mathserediffmail.com

Department of Mathematics and Applied Mathematics

UNIVERSITY OF THE FREE STATE

P. O. Box 339, 9300, BLOEMFonteIn, SOUTH AFricA

Email address: vetrikt@ufs.ac.za 